\title{
Leader-Based Consensus of Heterogeneous Nonlinear Multiagent Systems
}

\author{
Tairen Sun, ${ }^{1,2}$ Yongping Pan, ${ }^{3}$ and Haoyong $\mathrm{Yu}^{3}$ \\ ${ }^{1}$ School of Electrical and Information Engineering, Jiangsu University, Zhenjiang 212013, China \\ ${ }^{2}$ Key Laboratory of Autonomous Systems and Networked Control, Ministry of Education, \\ South China University of Technology, Guangzhou 510640, China \\ ${ }^{3}$ Department of Biomedical Engineering, National University of Singapore, Singapore 117575
}

Correspondence should be addressed to Tairen Sun; suntren@gmail.com

Received 17 March 2014; Revised 24 May 2014; Accepted 25 May 2014; Published 16 June 2014

Academic Editor: Leo Chen

Copyright (C) 2014 Tairen Sun et al. This is an open access article distributed under the Creative Commons Attribution License, which permits unrestricted use, distribution, and reproduction in any medium, provided the original work is properly cited.

This paper considers the leader-based consensus of heterogeneous multiple agents with nonlinear uncertain systems. Based on the information obtained from the following agents' neighbors, leader observers are designed by the following agents to estimate the leader's states and nonlinear dynamics. Then, to achieve leader-based consensus, adaptive distributed controllers are designed for the following agents to track the designed corresponding leader observers. The effectiveness of the leader observers and distributed consensus controllers are illustrated by formal proof and simulation results.

\section{Introduction}

In the past decades, cooperative control problems of multiagent have attracted more and more attention from researchers for its wide applications in many areas, such as formation control of mobile robots [1], monitoring [2], medical rescue [3], and environmental boundary tracking [4]. Consensus is a fundamental and important problem in the area of multiagent systems where a networked interaction protocol needs to be designed such that the final states of all agents converge to a common value.

Compared with the leaderless consensus in which the agents' states converge to a common constant value $[5,6]$, the states of multiagent can converge to a leader's dynamic state in leader-based consensus [7]. Though many results on leaderbased multiagent consensus have been obtained [7-14], most of the results considered agents with integrator or linear dynamics. However, most real agent systems are inherently nonlinear and uncertainty terms may exist in the systems. The consensus control in integrator or linear dynamics cannot be used directly in consensus of nonlinear agents. Recently, results [15-18] considered the leader-based consensus of homogeneous nonlinear agents with the assumption that the agents' nonlinear function satisfies the Lipschitz or likeLipchitz condition. The dynamical differences among agents are neglected in [15-18]. In the paper, we consider the leader-based consensus of heterogenous agents with different nonlinear dynamics.

NNs and fuzzy techniques are wildly used in control design for nonlinear uncertain systems, for their abilities in nonlinear function approximations $[19,20]$. In [21-25], the leader-based consensus controllers were designed for heterogeneous nonlinear uncertain multiagent systems. In the consensus control design, the leader's nonlinear dynamics was considered as a bounded disturbance and neural network- (NN-) based consensus control was designed for the following agents.

Distributed estimation via observer design for multiagent consensus is an important topic in the study of multiagent networks, with wide applications especially in sensor networks and robot networks, among many others [26]. Up to now, the results [26-31] have been obtained on multiagent consensus based on the distributed estimation via observer design. However, the considered agents in [26-31] are with 
integrator or linear dynamics. In this paper, the considered agents are with nonlinear uncertain dynamics and distributed NN-based leader observers are designed to estimate the leader's state and nonlinear dynamics. Then, to obtain the leader-based consensus, NN controllers are designed for the following agents to track the corresponding leader observers.

\section{Preliminaries}

2.1. Graph Theory and Some Notations. In this paper, we consider a networked system consisting of $N$ agents $v_{1}, v_{2}, \ldots, v_{N}$ and one leader $v_{0}$. The leader's system model and motion are independent of the other agents. Denote $G=(V, E)$ as a general digraph with the nonempty finite set of $N$ nodes or agents $V=\left\{v_{1}, v_{2}, \ldots, v_{N}\right\}$ and a set of edges or $\operatorname{arcs} E \subseteq V \times V$. Denote the connectivity matrix as $A=\left[a_{i j}\right]$ with $a_{i j}>0$ if $\left(v_{j}, v_{i}\right) \in E$ and $a_{i j}=0$ otherwise. Note $a_{i i}=0$. The set of neighbors of a node $v_{i}$ is $N_{i}=\left\{v_{j}:\left(v_{j}, v_{i}\right) \in E\right\}$, that is, the set of nodes with arcs incoming to $v_{i}$. Define the indegree matrix as a diagonal matrix $D=\operatorname{diag}\left\{d_{1}, d_{2}, \ldots, d_{N}\right\}$ with $d_{i}=\sum_{j \in N_{i}} a_{i j}$ for node $i$. Define the Laplacian matrix associated with graph $G$ as $L=D-A$.

Define an augmented graph as $\bar{G}=\{\bar{V}, \bar{E}\}$, where $\bar{V}=$ $\left\{v_{0}, v_{1}, \ldots, v_{N}\right\}$ and $\bar{E} \subseteq \bar{V} \times \bar{V}$. Denote the diagonal matrix $B=\operatorname{diag}\left\{b_{1}, b_{2}, \ldots, b_{N}\right\}$ as the leader adjacency matrix with $b_{i}=0$ if the $i$ th agent is a neighbor of the leader and $b_{i}=0$ otherwise.

Lemma 1 (see [22-24]). Let $L$ be irreducible and $B$ has at least one diagonal entry $b_{i}>0$. Then $L+B$ is a nonsingular $M-$ matrix. Define $q=\left[q_{1}, \ldots, q_{N}\right]^{T}=(L+B)^{-1} \underline{1}, P=\operatorname{diag}\left\{p_{i}\right\}=$ $\operatorname{diag}\left\{1 / q_{i}\right\}, Q=P(L+B)+(L+B)^{T} P$. Then $P$ and $Q$ are positive definite matrices.

2.2. Problem Statement. In the considered leader-based consensus, the leader's nonlinear dynamics is described as follows:

$$
\dot{x}_{0}=f_{0}\left(x_{0}\right)
$$

with $x_{0} \in R$ the state and $f_{0}\left(x_{0}\right)$ a piecewise continuous function of $x_{0}$.

Consider $N(N \geq 2)$ followed heterogenous agents with nonlinear uncertain dynamics described as follows:

$$
\dot{x}_{i}=f_{i}\left(x_{i}\right)+u_{i}, \quad i=1, \ldots, N
$$

where $x_{i}(t) \in R, u_{i}$ denote the state and the control input of the $i$ th agent, respectively; $f_{i}\left(x_{i}\right)$ is a nonlinear uncertain function, which is assumed to be continuous.

In this paper, we make the following assumptions: the following agent $i, i=1, \ldots, N$, can obtain its neighbors' states $x_{j}, j \in N_{i}$, and the leader's state estimation $\widehat{x}_{0 j}$, only some agents can obtain the leader's states $x_{0}$, and all the following agents do not know the leader's nonlinear dynamics.

The objective of this paper is to design controllers $u_{i}, i=$ $1, \ldots, N$, such that the consensus errors $\left|x_{i}-x_{0}\right|$ converge to a small neighborhood of zero.

\section{Leader-Based Consensus of Multiple Agents}

Since the nonlinear functions $f_{i}\left(x_{i}\right), f_{0}\left(x_{0}\right)$ are piecewise continuous and unknown to agent $i$, they can be estimated by agent $i$ using radial basis function (RBF) NNs on a compact set $\Omega_{i} \in R$ as follows:

$$
\begin{gathered}
\widehat{f}_{i}=W_{i}^{T} \phi_{i}\left(x_{i}\right), \\
\widehat{f}_{0 i}=W_{0 i}^{T} \varphi_{i}\left(\widehat{x}_{0 i}\right) .
\end{gathered}
$$

By ideal estimation the $f_{i}\left(x_{i}\right)$ and $f_{0}\left(x_{0}\right)$ can be written as

$$
\begin{array}{cl}
f_{i}\left(x_{i}\right)=W_{i}^{* T} \phi_{i}\left(x_{i}\right)+\epsilon_{i}\left(x_{i}\right), & \left|\epsilon_{i}\left(x_{i}\right)\right| \leq \epsilon_{i M}, \\
f_{0}\left(x_{0}\right)=W_{0 i}^{* T} \varphi_{i}\left(x_{0}\right)+\epsilon_{0 i}\left(x_{0}\right), & \left|\epsilon_{0 i}\left(x_{0}\right)\right| \leq \bar{\epsilon}_{i M},
\end{array}
$$

where $\phi_{i}\left(x_{i}\right) \in R^{n_{i}}$ and $\varphi_{i}\left(x_{0}\right) \in R^{m_{i}}$ are suitable basis sets of $n_{i}$ functions and $m_{i}$ functions, respectively; $W_{i}$ and $W_{0 i}$ are current estimation of ideal approximation weights $W_{i}^{*}$ and $W_{0 i}^{*}$, respectively; $\xi_{i}$ and $\zeta_{i}$ are bounds of ideal estimation errors $\epsilon_{i}\left(x_{i}\right)$ and $\epsilon_{0}\left(x_{0}\right)$, respectively; and $\widehat{x}_{0 i}$ is the estimation of $x_{0}$.

Remark 2. According to the definition of $\phi_{i}, \varphi_{i}, W_{i}, W_{0 i}$, it is easy to observe that there exist positive numbers $W_{M}, W_{0 M}$, $\epsilon_{M}$, and $\epsilon_{0 M}$, such that $\left\|W_{i}^{*}\right\| \leq W_{M},\left\|W_{0 i}^{*}\right\| \leq W_{0 M},\left|\epsilon_{i}\right| \leq \epsilon_{M}$, $\left|\epsilon_{0 i}\right| \leq \epsilon_{0 M}, i=1,2, \ldots, N$.

3.1. Observers for the Leader Agent. For agent $i$, the following NN-based observer is designed to estimate the leader's state and nonlinear dynamics:

$$
\dot{\hat{x}}_{0 i}=\widehat{f}_{0 i}-c \sum_{j \in N_{i}} a_{i j}\left(\widehat{x}_{0 i}-\widehat{x}_{0 j}\right)-c b_{i}\left(\widehat{x}_{0 i}-x_{0}\right) \text {. }
$$

Denote $\underline{x}_{0}=\left[x_{0}, x_{0}, \ldots, x_{0}\right]^{T} \in R^{N}, \underline{x}_{0}=\left[\widehat{x}_{01}, \ldots, \widehat{x}_{0 N}\right]^{T}$ $\in R^{N}, e_{0}=\underline{\hat{x}}_{0}-\underline{x}_{0}, e_{0 i}=\widehat{x}_{0 i}-x_{0}$. Taking time derivative of $e_{0}$ leads to

$$
\dot{e}_{0}=\widehat{f}_{0}-\underline{f}\left(x_{0}\right)-c(L+B) e_{0}
$$

with $\widehat{f}_{0}=\left[\widehat{f}_{01}, \widehat{f}_{02}, \ldots, \widehat{f}_{0 N}\right]^{T} \in R^{N}, \underline{f}\left(x_{0}\right)=$ $\left[f_{0}\left(x_{0}\right), \ldots, f_{0}\left(x_{0}\right)\right]^{T} \in R^{N}$.

From (4) and (6), $\widehat{f}_{0 i}-f_{0}\left(x_{0}\right)$ can be expressed as

$$
\begin{aligned}
\widehat{f}_{0 i}-f_{0}\left(x_{0}\right) & =W_{0 i}^{T} \varphi_{i}\left(\widehat{x}_{0 i}\right)-W_{0 i}^{* T} \varphi_{i}\left(x_{0}\right)-\epsilon_{0 i} \\
& =-W_{0 i}^{* T} \widetilde{\varphi}_{0 i}-\widetilde{W}_{0 i}^{T} \varphi_{i}\left(\widehat{x}_{0 i}\right)-\epsilon,
\end{aligned}
$$

where $\widetilde{W}_{0 i}=W_{0 i}^{*}-W_{0 i}$.

Remark 3. From Remark 2, we know that $-W_{0 i}^{* T} \widetilde{\varphi}_{0 i}-\epsilon_{0 i}, i=$ $1,2, \ldots, N$, are bounded. Then there exists a positive constant $\xi$, such that $\left|-W_{0 i}^{* T} \widetilde{\varphi}_{0 i}-\epsilon_{0 i}\right| \leq \xi$.

The following theorem illustrates the efficiency of the designed NN-observer. 
Theorem 4. Consider the leader and its following agents with the dynamics described by (1) and (2). If the NN weights in the observer (7) are updated by

$$
\dot{\widehat{W}}_{0 i}=-\dot{\widetilde{W}}_{0 i}=-F_{i}^{-1} e_{i} p_{i} \varphi_{i}\left(\widehat{x}_{0 i}\right)-k F_{i}^{-1} \widehat{W}_{0 i}
$$

then the estimation errors $e_{0 i}, \widetilde{W}_{0 i}, i=1,2, \ldots, N$, are uniformly ultimately bounded. Furthermore, the estimation error $e_{0 i}$ can be made arbitrarily small by a judicious choice of corresponding gains.

Proof. Consider the following Lyapunov function: $V_{0}=$ $(1 / 2) e_{0}^{T} P e_{0}+(1 / 2) \sum_{\mathrm{i}=1}^{N} \widetilde{W}_{0 i}^{T} F_{i} \widetilde{W}_{0 i}$. Take time derivative of $V_{0}$ and substitute (8) and (10). Consider

$$
\begin{aligned}
\dot{V}_{0}= & -c e_{0}^{T} P(L+B) e_{0}+e_{0}^{T} P\left(\widehat{f}_{0}-\underline{f}\left(x_{0}\right)\right)+\sum_{i=1}^{N} \widetilde{W}_{0 i}^{T} F_{i} \dot{\bar{W}}_{0 i} \\
= & -c e_{0}^{T}\left[P(L+B)+(L+B)^{T} P\right] e_{0} \\
& +\sum_{i=1}^{N} p_{i} e_{0 i}\left(-W_{0 i}^{* T} \widetilde{\varphi}_{0 i}-\epsilon_{0 i}\right) \\
& +\sum_{i=1}^{N} \widetilde{W}_{0 i}^{T}\left[F_{i} \dot{\widetilde{W}}_{0 i}-e_{i} p_{i} \varphi_{i}\left(\widehat{x}_{0 i}\right)\right] \\
\leq & -c e_{0}^{T} Q e_{0}+\xi \sum_{i=1}^{N} p_{i}\left|e_{0 i}\right|-k \sum_{i=1}^{N} \widetilde{W}_{0 i}^{T} \widetilde{W}_{0 i} \\
& +k \sum_{i=1}^{N}\left\|\widetilde{W}_{i}\right\| W_{0 M} .
\end{aligned}
$$

Since $p_{i}\left|e_{0 i}\right| \leq p_{\max }\left|e_{0 i}\right| \leq p_{\max }\left(\left(e_{0 i}^{2} / 2 \xi_{1}\right)+\left(\xi_{1} / 2\right)\right)$ with $p_{\max }=$ $\max \left\{p_{i}, i=1, \ldots, N\right\}$ and $\left\|\widetilde{W}_{i}\right\| \leq\left(1 / 2 \xi_{2}\right) \widetilde{W}_{i}^{T} \widetilde{W}_{i}+\left(\xi_{2} / 2\right)$ hold for arbitrary positive numbers $\xi_{1}, \xi_{2}$,

$$
\begin{aligned}
\dot{V}_{0} \leq & -\left(c \lambda_{\min }(Q)-\frac{\xi p_{\max }}{\left(2 \xi_{1}\right)}\right) e_{0}^{T} e_{0}-\left(k-\frac{k W_{0 M}}{\left(2 \xi_{2}\right)}\right) \sum_{i=1}^{N} \widetilde{W}_{i}^{T} \widetilde{W}_{i} \\
& +0.5 N\left(p_{\max } \xi_{1} \xi+\xi_{2} W_{0 M}\right) .
\end{aligned}
$$

From (12) we know that the derivative of $V_{0}$ is guaranteed to be less than zero, as long as $c \lambda_{\text {min }}(Q)-\xi p_{\max } /\left(2 \xi_{1}\right)>0, k-$ $k W_{0 M} /\left(2 \xi_{2}\right)>0$, and one of the following conditions holds:

$$
\begin{gathered}
\left\|e_{0}\right\| \geq \sqrt{\frac{0.5 N\left(p_{\max } \xi_{1} \xi+\xi_{2} W_{0 M}\right)}{c \lambda_{\min }(Q)-\xi_{p_{\max }} /\left(2 \xi_{1}\right)}}, \\
\sum_{i=1}^{N} \widetilde{W}_{0 i}^{T} \widetilde{W}_{0 i} \geq \frac{0.5 N\left(p_{\max } \xi_{1} \xi+\xi_{2} W_{0 M}\right)}{k-k W_{0 M} /\left(2 \xi_{2}\right)} .
\end{gathered}
$$

Therefore $\dot{V}_{0}$ is negative outside a compact set. According to the standard Lyapunov theory extension, the estimation errors $e_{0 i}, \widetilde{W}_{0 i}, i=1,2, \ldots, N$, are uniformly ultimately bounded. Furthermore, the estimation error $e_{0 i}$ can be made arbitrarily small by a judicious choice of corresponding gains.
3.2. Leader-Based Consensus Control Design. Since the effectiveness of the leader observers's estimation of the leader's state and dynamics, the leader-based consensus problem can be solved if some controllers are designed for the following agents to track the designed observers. In this part, NN-based controllers are designed for the agents $i, i=1,2, \ldots, N$, to track the corresponding observers.

Denote $e_{i}=x_{i}-\widehat{x}_{0 i}$. Take time derivative of $e_{i}$ and substitute (2) and (8). Consider

$$
\dot{e}_{i}=f_{i}\left(x_{i}\right)+u_{i}-\widehat{f}_{0 i}+c \sum_{j \in N_{i}} a_{i j}\left(\widehat{x}_{0 i}-\widehat{x}_{0 j}\right)+c b_{i}\left(\widehat{x}_{0 i}-x_{0}\right) \text {. }
$$

If the control law $u_{i}$ is designed as

$$
u_{i}=-l_{\mathrm{i}} e_{i}-\widehat{f}_{i}+\widehat{f}_{0 i}-c \sum_{j \in N_{i}} a_{i j}\left(\widehat{x}_{0 i}-\widehat{x}_{0 j}\right)-c b_{i}\left(\widehat{x}_{0 i}-x_{0}\right)
$$

then we have

$$
\begin{aligned}
\dot{e}_{i} & =-l_{i} e_{i}+W_{i}^{* T} \phi_{i}\left(x_{i}\right)-W_{i}^{T} \phi_{i}\left(x_{i}\right)+\epsilon_{i} \\
& =-l_{i} e_{i}+\widetilde{W}_{i}^{T} \phi\left(x_{i}\right)+\epsilon_{i} .
\end{aligned}
$$

Based on the above analysis, we have the following result.

Theorem 5. Consider the distributed system (1) and the leader (2). If the control law for the following agents is designed as (15) and the NN weights $W_{i}$ are updated as

$$
\dot{W}_{i}=-\dot{\widetilde{W}}_{i}=\bar{F}_{i}^{-1} \phi_{i}\left(x_{i}\right) e_{i},
$$

then tracking errors $e_{i}, i=1,2, \ldots, N$, are uniformly ultimately bounded and can be made arbitrarily small by appropriate choice of corresponding gains; that is, the leaderbased consensus is achieved.

Proof. Consider the candidate Lyapunov function $V_{1}=$ $(1 / 2) e_{i}^{2}+(1 / 2) \widetilde{W}_{i}^{T} \bar{F}_{i} \widetilde{W}_{i}$. Take time derivative of $V_{1}$ and substitute (16)-(17). Consider

$$
\begin{aligned}
\dot{V}_{1} & =e_{i}\left(-l_{i} e_{i}+\widetilde{W}_{i}^{T} \phi\left(x_{i}\right)+\epsilon_{i}\right)+\widetilde{W}_{i}^{T} \bar{F}_{i} \dot{\widetilde{W}}_{i} \\
& =-l_{i} e_{i}^{2}+e_{i} \epsilon_{i}+\widetilde{W}_{i}^{T}\left(\bar{F}_{i} \dot{\bar{W}}_{i}+\phi_{i}\left(x_{i}\right) e_{i}\right) \\
& \leq-l_{i} e_{i}^{2}+\left|e_{i}\right| \epsilon_{N} .
\end{aligned}
$$

Since $\left|e_{i}\right| \epsilon_{N} \leq\left(e_{i}^{2} / 2\right)+\left(\epsilon_{N}^{2} / 2\right)$ holds,

$$
\dot{V}_{1} \leq-\left(l_{i}-\frac{1}{2}\right) e_{i}^{2}+\frac{\epsilon_{N}^{2}}{2} .
$$

From (19) we know that $\dot{V}_{1}$ is negative, as long as $l_{i}-$ $(1 / 2)>0$ and $\left|e_{i}\right| \geq\left(\epsilon_{N} / \sqrt{2 l_{i}-1}\right)$. Therefore, according to the standard Lyapunov theory extension, the tracking errors $e_{i}$ are uniformly ultimately bounded and can be made arbitrarily small by a judicious choice of corresponding gains. 
Since $x_{i}-x_{0}=x_{i}-\widehat{x}_{0 i}+\widehat{x}_{0 i}-x_{0}, i=1,2, \ldots, N$, from Theorem 4 and the uniformly ultimately boundedness of $e_{i}=x_{i}-\widehat{x}_{0 i}, i=1,2, \ldots, N$, we can conclude that the consensus errors $x_{i}-x_{0}, i=1,2, \ldots, N$, are uniformly bounded and can be made arbitrarily small by appropriate choice of corresponding gains.

\section{Simulation Results}

Consider a group of nonlinear uncertain agents composed of a leader agent 0 and four following agents 1,2,3,4 described in Figure 1. Let the dynamics of the agents be

$$
\begin{gathered}
\dot{x}_{0}=\sin x_{0} \\
\dot{x}_{1}=\cos x_{1}+u_{1} \\
\dot{x}_{2}=2 x_{2}+\sin x_{2}+u_{2} \\
\dot{x}_{4}=\cos x_{4}+u_{4} .
\end{gathered}
$$

Simulations are carried out on Pentinum(R) Dual-Core CPU and Matlab 2008b environments. In the simulation, the initial values of the five agents are $x_{0}(0)=1, x_{1}(0)=2, x_{2}(0)=4$, $x_{3}(0)=3$, and $x_{4}(0)=1$ and the $\mathrm{NN}$ weights and the observers are initialized to be zero vectors or zero. Choose the design parameters as $k=3, F_{i}^{-1}=100, c=5, l_{1}=5$, $l_{2}=8, l_{3}=9$, and $l_{4}=5$. The simulation results are present in Figures 2-4, where leader-based consensus errors $x_{0}-x_{i}$, $i=1,2,3,4$, are presented in Figure 2, and the estimation errors $x_{0}-\widehat{x}_{0 i}, i=1,2,3,4$, in the leader observers are presented in Figures 3 and 4 describing the tracking errors $x_{i}-x_{0 i}, i=1,2,3,4$, between the following agents and their corresponding observers. From the simulation results in Figures 2-4, the errors $x_{0}-x_{i}, x_{0}-\widehat{x}_{0 i}$, and $x_{i}-x_{0 i}, i=1,2,3,4$, converge to very small neighborhoods of zero after 3 seconds. So, we can conclude that the leader observers (7) are effective to estimate the leader's states and nonlinear dynamics and the leader-based consensus of multiple nonlinear uncertain agents can be achieved under the controllers (15).

\section{Conclusions}

This paper addressed the NN-observer-based leader-following consensus of heterogeneous multiagent systems with nonlinear uncertain dynamics. NN-based leader observers were designed to estimate the leader's state and nonlinear dynamics. Then NN-based controllers were designed for the following agents to track the corresponding leader observer so that leader-based consensus can be achieved. The effectiveness of the consensus construction method was illustrated by theoretical analysis and simulation results.

In this paper, the considered leader was with nonlinear time-invariant system. The observer-based leader-following consensus of nonlinear uncertain systems, in which the leader is with nonlinear time-varying system, needs to be considered in the future.

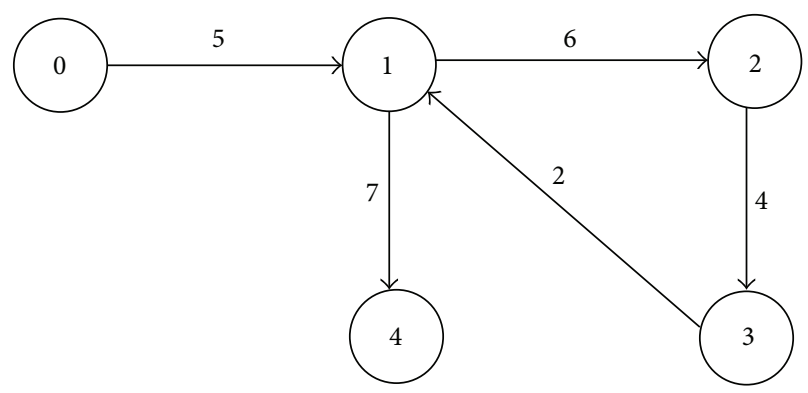

Figure 1: Communication topology of five agents.

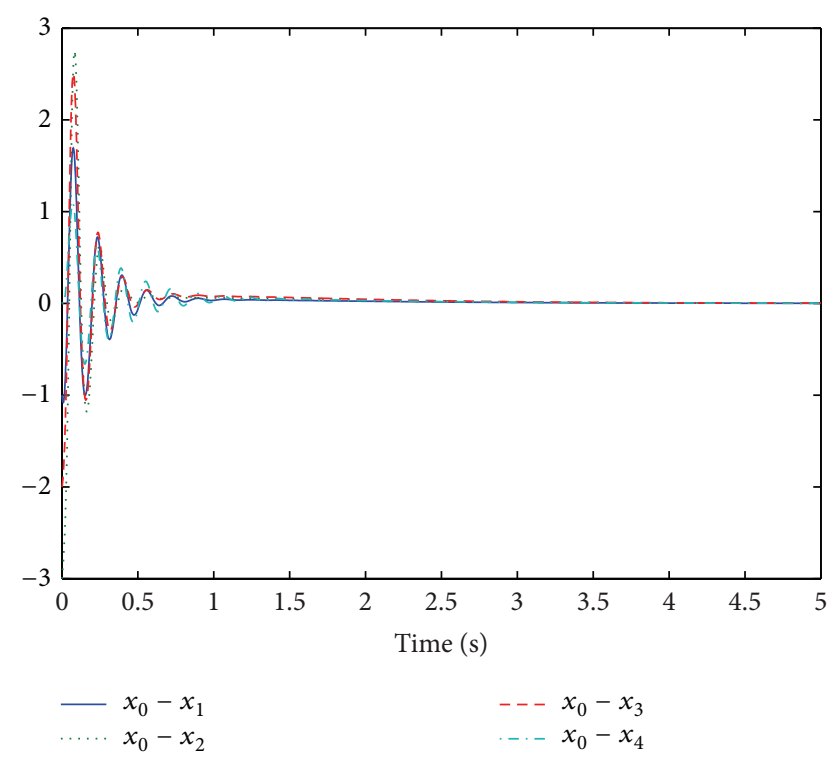

Figure 2: Leader-based consensus errors $x_{0}-x_{i}, i=1,2,3,4$.

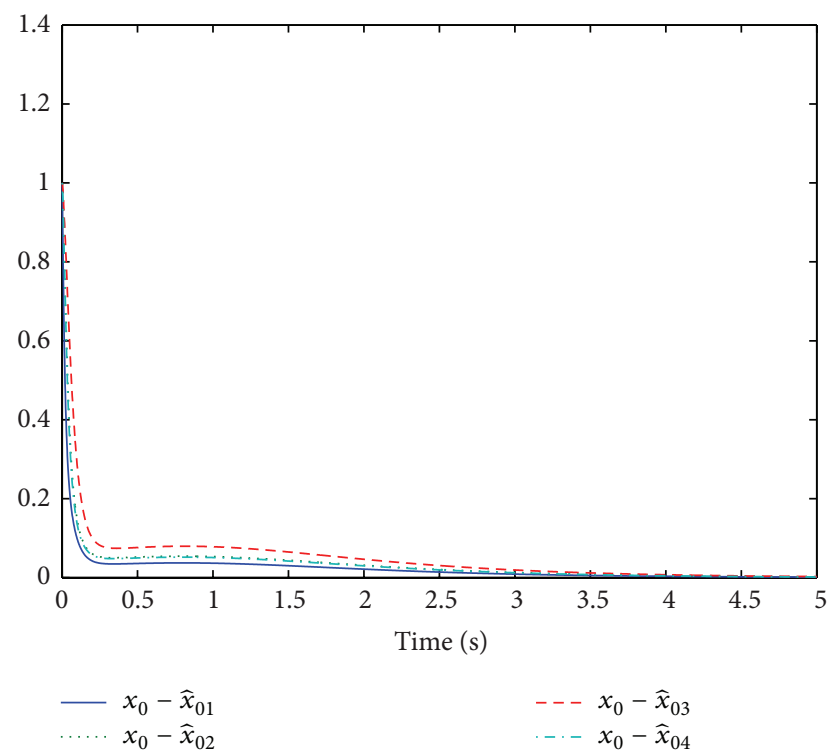

FIgURE 3: Leader observers' estimation errors $x_{0}-\widehat{x}_{0 i}, i=1,2,3,4$. 


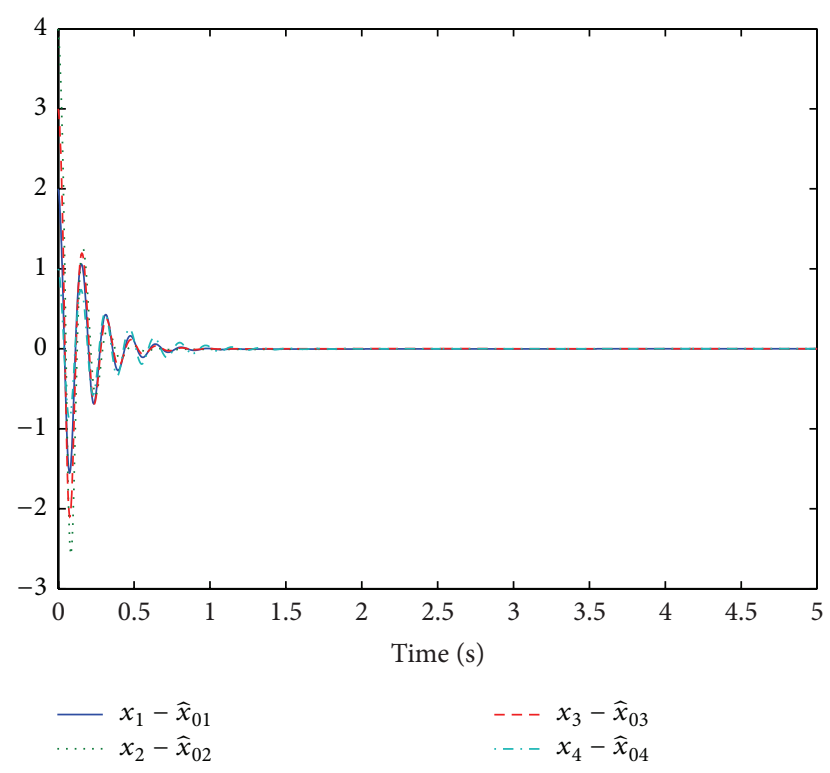

FIgURE 4: The tracking errors $x_{i}-x_{0 i}, i=1,2,3,4$, between the following agents and their corresponding observers.

\section{Conflict of Interests}

The authors declare that there is no conflict of interests regarding the publication of this paper.

\section{Acknowledgments}

This work was supported by the National Natural Science Foundation of China (61304073), the Natural Science Foundation of Jiangsu Province (BK20130536 and BK20130533), China Postdoctoral Science Foundation (2013M541615 and 2013M540421), Postdoctoral Science Foundation of Jiangsu Province (1301044B), and the Priority Academic Program Development of Jiangsu Higher Education Institutions.

\section{References}

[1] T. Sun, F. Liu, H. Pei, and Y. He, "Observer-based adaptive leader-following formation control for non-holonomic mobile robots," IET Control Theory \& Applications, vol. 6, no. 18, pp. 2835-2841, 2012.

[2] J. T. Feddema, C. Lewis, and D. A. Schoenwald, "Decentralized control of cooperative robotic vehicles: theory and application," IEEE Transactions on Robotics and Automation, vol. 18, no. 5, pp. 852-864, 2002.

[3] J. S. Jennings, G. Whelan, and W. F. Evans, "Cooperative search and rescue with a team of mobile robots," in Proceedings of the 8th International Conference on Advanced Robotics (ICAR '97), pp. 193-200, July 1997.

[4] T. Sun, H. Pei, Y. Pan, and C. Zhang, "Robust adaptive neutral network control for environmental boundary tracking by mobile robots," International Journal of Robust and Nonlinear Control, vol. 23, no. 2, pp. 123-136, 2013.

[5] Z.-G. Hou, L. Cheng, and M. Tan, "Decentralized robust adaptive control for the multiagent system consensus problem using neural networks," IEEE Transactions on Systems, Man, and Cybernetics, Part B, vol. 39, no. 3, pp. 636-647, 2009.

[6] Z.-X. Liu and Z.-Q. Chen, "Discarded consensus of network of agents with state constraint," IEEE Transactions on Automatic Control, vol. 57, no. 11, pp. 2869-2874, 2012.

[7] L. Cheng, Y. Wang, Z.-G. Hou, M. Tan, and Z. Cao, "Sampled-data based average consensus of second-order integral multi-agent systems: switching topologies and communication noises," Automatica, vol. 49, no. 5, pp. 1458-1464, 2013.

[8] S. Li, H. Du, and X. Lin, "Finite-time consensus algorithm for multi-agent systems with double-integrator dynamics," Automatica, vol. 47, no. 8, pp. 1706-1712, 2011.

[9] F. Jiang, L. Wang, and G. Xie, "Consensus of high-order dynamic multi-agent systems with switching topology and time-varying delays," Journal of Control Theory and Applications, vol. 8, no. 1, pp. 52-60, 2010.

[10] K. Liu, G. Xie, and L. Wang, "Consensus for multi-agent systems under double integrator dynamics with time-varying communication delays," International Journal of Robust and Nonlinear Control, vol. 22, no. 17, pp. 1881-1898, 2012.

[11] F. Xiao and T. Chen, "Sampled-data consensus for multiple double integrators with arbitrary sampling," IEEE Transactions on Automatic Control, vol. 57, no. 12, pp. 3230-3235, 2012.

[12] Y. Zhang and Y.-P. Tian, "Allowable sampling period for consensus control of multiple general linear dynamical agents in random networks," International Journal of Control, vol. 83, no. 11, pp. 2368-2377, 2010.

[13] Y. Gao and L. Wang, "Sampled-data based consensus of continuous-time multi-agent systems with time-varying topology," IEEE Transactions on Automatic Control, vol. 56, no. 5, pp. 1226-1231, 2011.

[14] Y. Liu and Y. Jia, "Robust Ho consensus control of uncertain multi-agent systems with time delays," International Journal of Control, Automation and Systems, vol. 9, no. 6, pp. 1086-1094, 2011.

[15] Y. Zhao, Z. Li, and Z. Duan, "Distributed consensus tracking of multi-agent systems with nonlinear dynamics under a reference leader," International Journal of Control, vol. 86, no. 10, pp. 18591869, 2013.

[16] Y. Hu, H. Su, and J. Lam, "Adaptive consensus with a virtual leader of multiple agents governed by locally Lipschitz nonlinearity," International Journal of Robust and Nonlinear Control, vol. 23, no. 9, pp. 978-990, 2013.

[17] W. Yu, W. Ren, W. X. Zheng, G. Chen, and J. Lü, "Distributed control gains design for consensus in multi-agent systems with second-order nonlinear dynamics," Automatica, vol. 49, no. 7, pp. 2107-2115, 2013.

[18] Y. Zhao, B. Li, J. Qin, H. Gao, and H. R. Karimi, "Consensus and synchronization of nonlinear systems based on a novel fuzzy model," IEEE Transactions on Cybernetics, vol. 43, no. 6, pp. 2157-2169, 2013.

[19] Y. Pan, M. J. Er, D. Huang, and Q. Wang, "Adaptive fuzzy control with guaranteed convergence of optimal approximation error," IEEE Transactions on Fuzzy Systems, vol. 19, no. 5, pp. 807-818, 2011.

[20] Y. P. Pan, H. Y. Yu, and M. J. Er, "Adaptive neural PD control with semiglobal asymptotic stabilization guarantee," IEEE Transactions on Neural Networks and Learning Systems, 2014.

[21] Z. Peng, D. Wang, G. Sun, and H. Wang, "Distributed cooperative stabilisation of continuous-time uncertain nonlinear multiagent systems," International Journal of Systems Science, vol. 44, pp. 1-11, 2013. 
[22] A. Das and F. L. Lewis, "Distributed adaptive control for synchronization of unknown nonlinear networked systems," Automatica, vol. 46, no. 12, pp. 2014-2021, 2010.

[23] A. Das and F. L. Lewis, "Cooperative adaptive control for synchronization of second-order systems with unknown nonlinearities," International Journal of Robust and Nonlinear Control, vol. 21, no. 13, pp. 1509-1524, 2011.

[24] H. Zhang and F. L. Lewis, "Adaptive cooperative tracking control of higher-order nonlinear systems with unknown dynamics," Automatica, vol. 48, no. 7, pp. 1432-1439, 2012.

[25] Z. Peng, D. Wang, H. Zhang, G. Sun, and H. Wang, "Distributed model reference adaptive control for cooperative tracking of uncertain dynamical multi-agent systems," IET Control Theory \& Applications, vol. 7, no. 8, pp. 1079-1087, 2013.

[26] Y. Hong, G. Chen, and L. Bushnell, "Distributed observers design for leader-following control of multi-agent networks," Automatica, vol. 44, no. 3, pp. 846-850, 2008.

[27] H. Zhang, F. L. Lewis, and A. Das, "Optimal design for synchronization of cooperative systems: state feedback, observer and output feedback," IEEE Transactions on Automatic Control, vol. 56, no. 8, pp. 1948-1952, 2011.

[28] Z. Li, X. Liu, P. Lin, and W. Ren, "Consensus of linear multiagent systems with reduced-order observer-based protocols," Systems \& Control Letters, vol. 60, no. 7, pp. 510-516, 2011.

[29] K. Hengster-Movric and F. Lewis, "Cooperative observers and regulators for discrete-time multiagent systems," International Journal of Robust and Nonlinear Control, vol. 23, no. 14, pp. 1545-1562, 2013.

[30] Z. Meng and Z. Lin, "On distributed finite-time observer design and finite-time coordinated tracking of multiple double integrator systems via local interactions," International Journal of Robust and Nonlinear Control, 2013.

[31] B. Zhou, C. Xu, and G. Duan, "Distributed and truncated reduced-order observer based ouput feedback consensus of multi-agent systems," IEEE Transactions on Automatic Control, 2014. 


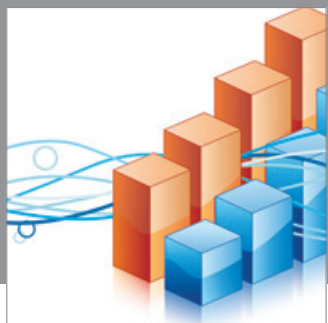

Advances in

Operations Research

mansans

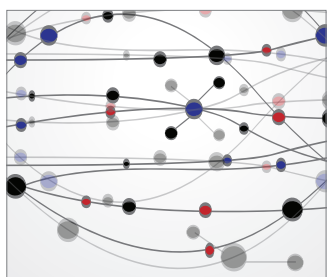

The Scientific World Journal
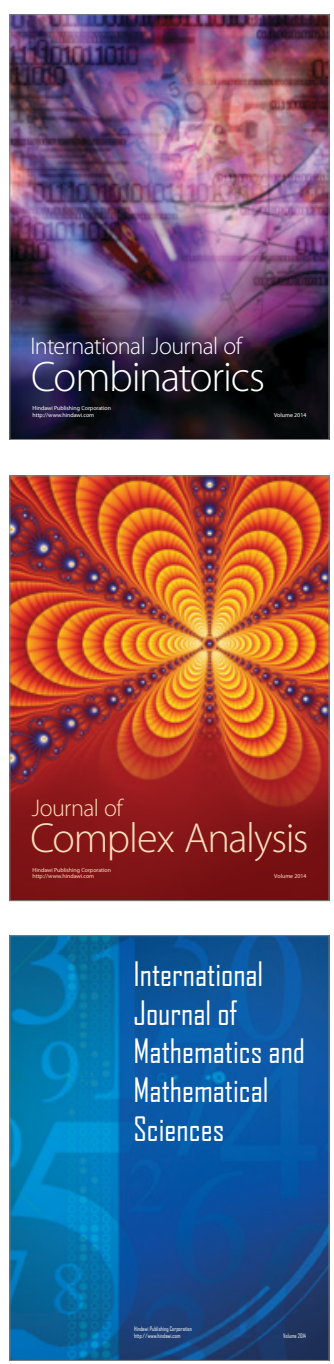
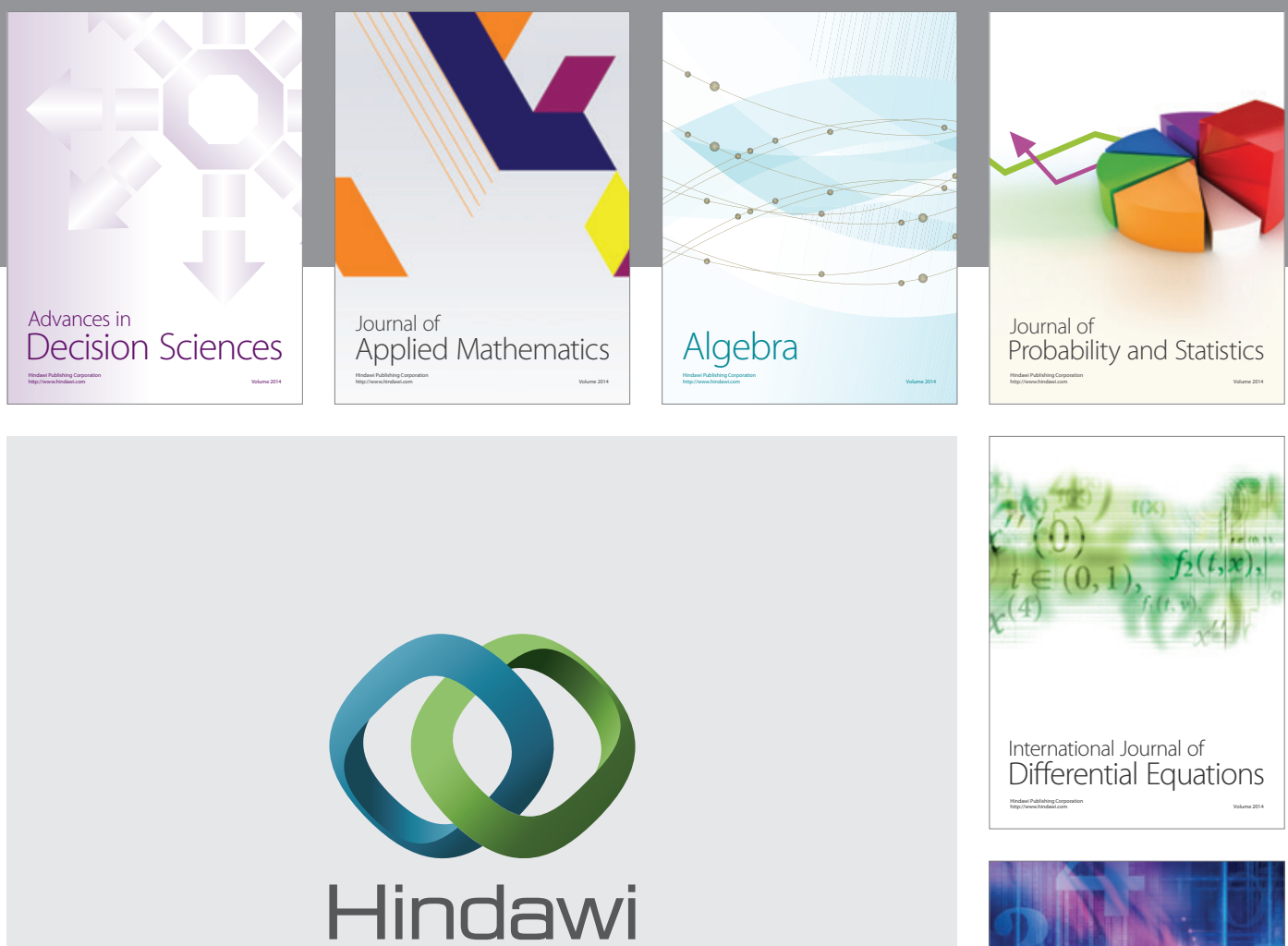

Submit your manuscripts at http://www.hindawi.com
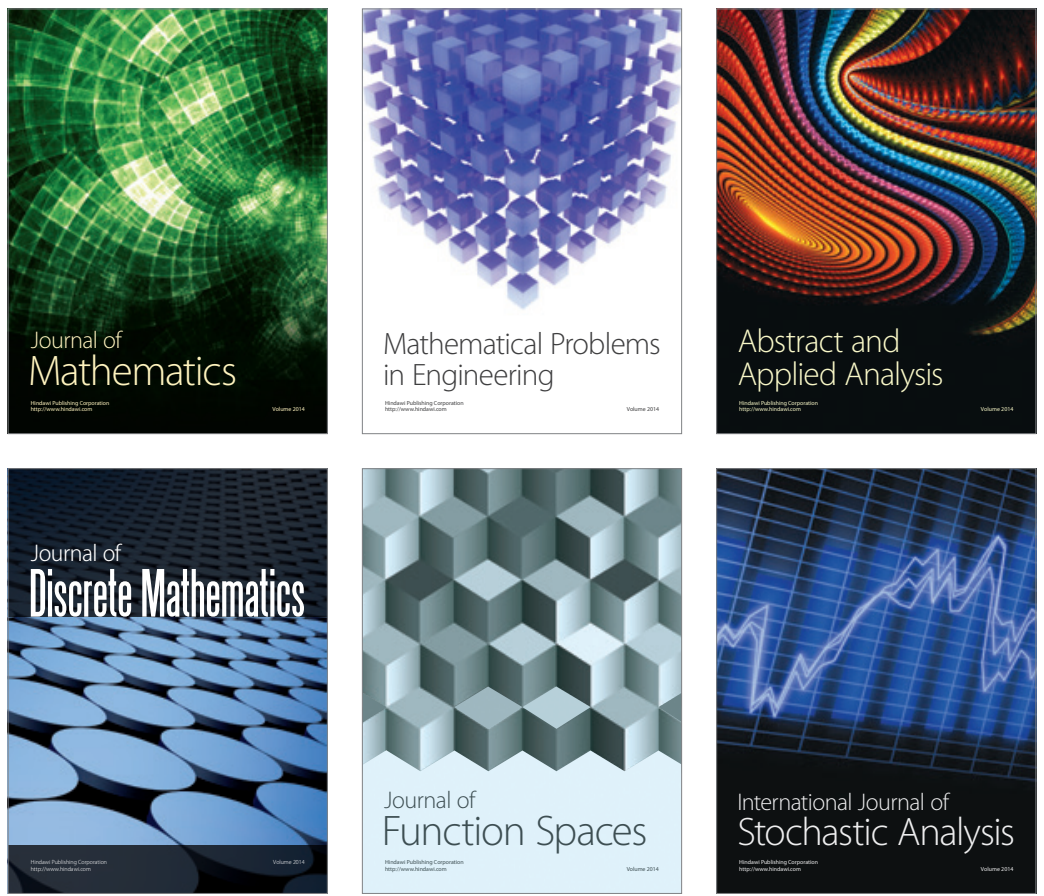

Journal of

Function Spaces

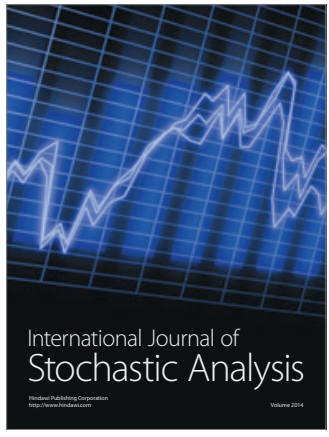

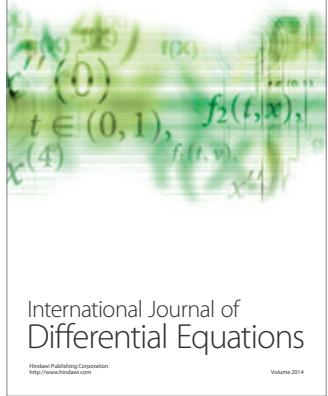
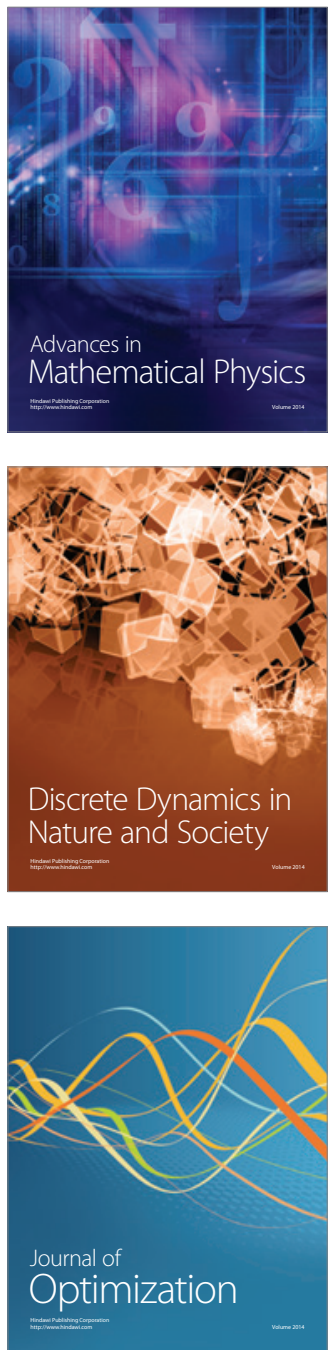